\title{
Bicuspid aortic valve aortopathy in the era of fake news: A clinician's perspective on the truth
}

\author{
Hector I. Michelena, MD
}

From the Department of Cardiovascular Diseases, Mayo Clinic, Rochester, Minn.

Disclosures: Author has nothing to disclose with regard to commercial support.

Received for publication March 21, 2018; accepted for publication March 22, 2018; available ahead of print April $18,2018$.

Address for reprints: Hector I. Michelena, MD, Department of Cardiovascular Diseases, Mayo Clinic, 200 First St SW, Rochester, MN 55905 (E-mail: michelena.hector@mayo.edu).

J Thorac Cardiovasc Surg 2018;156:16-7

$0022-5223 / \$ 36.00$

Copyright (C) 2018 by The American Association for Thoracic Surgery

https://doi.org/10.1016/j.jtcvs.2018.03.076

The researcher's Holy Grail in bicuspid aortopathy is to understand and avert aortic dissection, a deadly but infrequent occurrence. ${ }^{1,2}$ Consequently, bicuspid aortopathy researchers are faced with 2 inevitabilities: (1) the necessary use of surrogate events, such as aorta dilatation and elective aorta surgery; and (2) the obligatory contentment with retrospective, observational, and pseudo-natural history studies. Retrospective because events are scarce and occur late (prospective studies would be highly demanding), observational because there rarely is equipoise in dissection-preventing procedures that could empower randomization, and pseudo-natural history because some degree of intervention is permitted (if rational) in light of the seriousness of the Holy Grail event. Such is the case of the study by Hui and colleagues, ${ }^{3}$ one of the largest of its kind to date, in which 406 consecutive nonsyndromic patients (269 with bicuspid and 137 with tricuspid aortic valves) who underwent aortic valve replacement (AVR) and supracoronary ascending aorta replacement (AAR) were followed $8 \pm 5$ years for root events ( $\sim 80 \%$ complete follow-up), yet one third of them underwent concomitant noncoronary sinus replacement at baseline surgery per the surgeons' judgment. This should not be surprising for 2 reasons: (1) the echocardiographic bidimensional long-axis measurement of the root, particularly in patients with bicuspid valves, systematically underestimates its true measurement ${ }^{4}$ and imagers rarely report root asymmetry; and (2) no imaging modality can determine the quality of the aortic tissue; thus, the surgeon may decide to partially address a tissue-deficient root even if not significantly dilated. Notwithstanding, Hui and colleagues $^{3}$ showed that within midterm follow-up of nonsyndromic patients with a nondilated root who underwent AVR-AAR for predominant valve stenosis, no patient developed significant root dilation or root dissection at follow-up. These findings are important in light of 2 surgical truths: (1) Cardiopulmonary bypass time is always running, and (2) coronary artery reimplantation may be

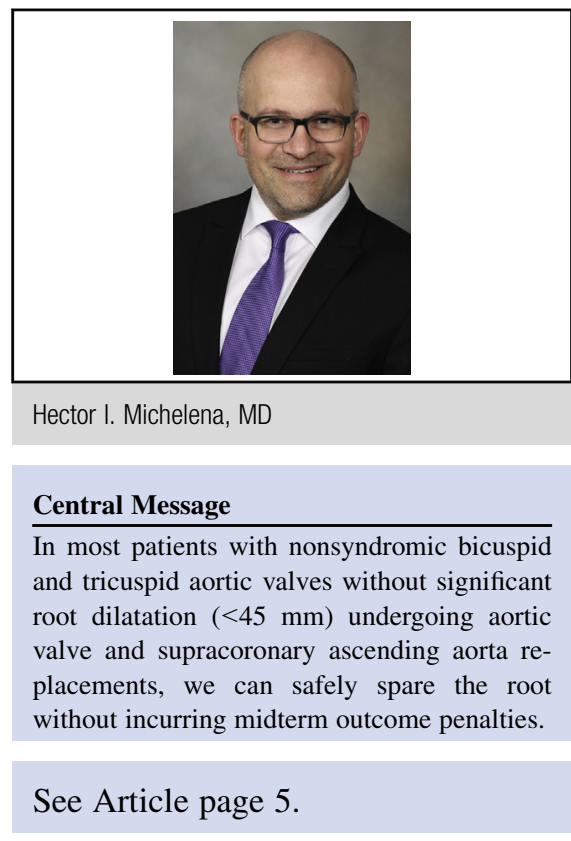

technically challenging. In addition, albeit limited imaging follow-up ( $\sim 65 \%$ of patients), the annualized postsurgical root dilation rate, although not negligible, was certainly no different than previously reported $(0.4-0.6 \mathrm{~mm} / \mathrm{y})^{5}$ and not different between patients with bicuspid valves and patients with tricuspid valves. Furthermore, the cumulative rates of aortic reoperation were also similar between those with bicuspid and tricuspid valves.

In an era of misinformation and disregard for expertise, we have more than ever the responsibility to build an approximation to the truth that is evidence based and bias free. To decipher any truth from available evidence in aortopathy research, we must understand some premises: (1) We cannot infer causality from observational studies (ie, whether noncoronary sinus plasty or AVR-AAR stabilizes the root remains unknown), but we can glimpse at the outcomes of clinical practice in a systematic way and uncover associations and their strength. (2) Positive observational results may suggest the possible benefit of a procedure, but more important, they are a faithful reflection of the clinical/surgical judgment of the physicians and the institutional experience, so we must carefully look at the algorithms and techniques used (ie, how patients were chosen for what surgery). (3) When ascertaining a deadly but uncommon event such as aortic dissection/rupture, any loss to follow-up may well be that event (ie, incomplete follow-up is a major problem with these studies). (4) Any 
conclusion can be applied only to patients well represented in the study (ie, this study applies only to nonsyndromic patients undergoing AVR-AAR with nondilated roots).

The past 2 decades have seen a tendency to be "more aggressive" with the bicuspid aorta with little or no evidence that it will improve outcomes. Hui and colleagues' ${ }^{3}$ findings provide support to the concept that the clinical behavior of nonsyndromic bicuspid aortopathy is no different than its tricuspid counterpart, and both are considerably more benign than syndromic aortopathies (ie, Marfan syndrome). ${ }^{6-8}$ The authors' findings also contribute to a growing body of observational literature that suggests we can safely spare the root without incurring midterm outcome penalties in most nonsyndromic patients with bicuspid and tricuspid valves undergoing AVR-AAR who have no significant root dilatation $(<45 \mathrm{~mm}){ }^{9-13}$ Because the most common bicuspid complication is valvular dysfunction requiring $\mathrm{AVR},{ }^{5}$ and bicuspid aortopathy manifests most commonly with tubular ascending aorta dilatation $(60 \%-70 \%$ vs $\sim 25 \%$ root $),{ }^{5,8}$ where the tubular ascending aorta exhibits the fastest dilatation rate, ${ }^{8}$ these emerging concepts apply to the majority of patients with bicuspid valves facing surgery. The relevance of noncoronary sinus repair and "root phenotype" features (male sex, younger age, root dilation and aortic regurgitation) $)^{5}$ in these patients with bicuspid valves remains unknown. Interestingly, however, Milewski and colleagues ${ }^{11}$ recently reported no adverse root outcomes (median follow-up $\sim 3.5$ years) without coronary sinus plasty in patients with bicuspid valves with pure aortic regurgitation and some root dilatation $(40-45 \mathrm{~mm})$ undergoing AVR-AAR, yet appropriately cautioned on the need for further follow-up of these patients. Clearly, the pursuit of long-term complete clinical follow-up of these studies is critical.

Finally, it is important to recognize that despite the purported annular and sinotubular junction stabilization (AVR-AAR), the root does continues to dilate. ${ }^{3,9}$ In light of this and a persistently elusive Holy Grail, whether a portion of the thoracic aorta was replaced or not, patients with bicuspid valves post-AVR require continued interval thoracic aorta imaging surveillance. Building an approximation to the truth in bicuspid aortopathy remains a work in progress.

\section{References}

1. Michelena HI, Desjardins VA, Avierinos JF, Russo A, Nkomo VT, Sundt TM, et al. Natural history of asymptomatic patients with normally functioning or minimally dysfunctional bicuspid aortic valve in the community. Circulation 2008;117:2776-84

2. Michelena HI, Khanna AD, Mahoney D, Margaryan E, Topilsky Y, Suri RM, et al. Incidence of aortic complications in patients with bicuspid aortic valves. JAMA. 2011;306:1104-12.

3. Hui SK, Fan C-PS, Christie S, Feindel CM, David TE, Ouzounian M. The aortic root does not dilate over time after replacement of the aortic valve and ascending aorta in patients with bicuspid or tricuspid aortic valves. $J$ Thorac Cardiovasc Surg. 2018;156:5-13.e1.

4. Park JY, Foley TA, Bonnichsen CR, Maurer MJ, Goergen KM, Nkomo VT, et al. Transthoracic echocardiography versus computed tomography for ascending aortic measurements in patients with bicuspid aortic valve. J Am Soc Echocardiogr. 2017;30:625-35.

5. Michelena HI, Prakash SK, Della Corte A, Bissell MM, Anavekar N, Mathieu P, et al. Bicuspid aortic valve: identifying knowledge gaps and rising to the challenge from the international bicuspid aortic valve consortium (Bavcon). Circulation. 2014;129:2691-704.

6. Weinsaft JW, Devereux RB, Preiss LR, Feher A, Roman MJ, Basson CT, et al. Aortic dissection in patients with genetically mediated aneurysms: incidence and predictors in the Gentac registry. J Am Coll Cardiol. 2016;67: 2744-54.

7. Itagaki S, Chikwe JP, Chiang YP, Egorova NN, Adams DH. Long-term risk for aortic complications after aortic valve replacement in patients with bicuspid aortic valve versus Marfan syndrome. J Am Coll Cardiol. 2015; 65:2363-9.

8. Detaint D, Michelena HI, Nkomo VT, Vahanian A, Jondeau G, Sarano ME. Aortic dilatation patterns and rates in adults with bicuspid aortic valves: a comparative study with Marfan syndrome and degenerative aortopathy. Heart 2014;100:126-34.

9. Peterss S, Bhandari R, Rizzo JA, Fang H, Kuzmik GA, Ziganshin BA, et al. The aortic root: natural history after root-sparing ascending replacement in nonsyndromic aneurysmal patients. Ann Thorac Surg. 2017;103:828-33.

10. Wang Y, Lin Y, Yin K, Zhu K, Yang Z, Sun Y, et al. Should sinus of Valsalva be preserved in patients with bicuspid aortic valve and aortic dilation? J Thorac Dis. 2017:9:3148-53.

11. Milewski RK, Habertheuer A, Bavaria JE, Siki M, Szeto WY, Krause E, et al. Fate of remnant sinuses of Valsalva in patients with bicuspid and trileaflet valves undergoing aortic valve, ascending aorta, and aortic arch replacement. J Thorac Cardiovasc Surg. 2017;154:421-32.

12. Vendramin I, Meneguzzi M, Sponga S, Deroma L, Cimarosti R, Lutman C, et al. Bicuspid aortic valve disease and ascending aortic aneurysm: should an aortic root replacement be mandatory? Eur J Cardiothorac Surg. 2016;49: 103-9.

13. Park CB, Greason KL, Suri RM, Michelena HI, Schaff HV, Sundt TM III. Fate of nonreplaced sinuses of Valsalva in bicuspid aortic valve disease. J Thorac Cardiovasc Surg. 2011;142:278-84. 